\title{
Quantitative EDX and EELS Elemental Mapping at Atomic Resolution
}

\author{
G. Kothleitner ${ }^{1}$, M. J. Neish ${ }^{2}$, N. R. Lugg ${ }^{2,4}$, S. D. Findlay ${ }^{3}$, W. Grogger ${ }^{1}$, F. Hofer ${ }^{1}$, and L. J. Allen ${ }^{2}$ \\ ${ }^{1}$ Institute f. Electron Microscopy (FELMI), Graz University of Technology and Centre f. Electron \\ Microscopy (ZFE), Graz, Steyrergasse 17, 8010 Graz, Austria \\ ${ }^{2}$ School of Physics, University of Melbourne, Parkville, Victoria 3010, Australia \\ ${ }^{3}$ School of Physics, Monash University, Clayton, Victoria 3800, Australia \\ ${ }^{4}$ Institute of Engineering Innovation, The University of Tokyo, Tokyo 113-8656, Japan
}

$\mathrm{C}_{\mathrm{S}}$-aberration correction of electron probes as used in a scanning transmission electron microscope (STEM) has enabled numerous applications, showing atomic resolution analysis from interesting materials science samples [1,2,3]. The high-angle annular dark-field Z-contrast imaging technique has long been widely used, but only recently has become quantitative, in the sense that counting atoms and correlating their occurrence in the unit cell / structure has become possible $[4,5]$.

This progress in STEM imaging for a long time remained unaccompanied by equivalent improvements in the equipment needed to augment such measurements with analytical EELS (electron energy loss spectroscopy) and EDXS (energy dispersive x-ray spectroscopy) data. However, the latest innovations such as collection-efficient solid state X-ray detectors and EELS spectrometers for the simultaneous recording of both the low-loss and the core-loss region have made analytical investigations much more powerful and reliable [6,7]. In order to combine both techniques and harness their respective potentials for light- and heavy-element quantitative atomic resolution analysis, we have set up a powerful hardware configuration (GIF Quantum, Bruker / FEI Super-X detector, Cs-probe corrected Titan, operated under de-embedded 64bit DigitalMicrograph and driven by DigiScan) and, secondly, have implemented an analysis concept which reduces the need for estimates on most of the parameters needed for quantitative EDXS and EELS (like sample densities, absolute thicknesses, theoretical ionization cross-sections etc.) [8].

Even then, putting meaningful quantitative figures on atomic resolution maps in terms of volumetric concentrations requires detailed theoretical simulations, as the complex physics of scattering unlinks the observed analytical intensities from the projected atomic positions. With the use of the so-called quantum excitation of phonons (QEP) model [9], a calculation of the underlying elastic and thermal diffuse scattering (TDS) is possible and quantitative comparisons between experiment and quantum mechanical calculations for both EDXS and EELS can be made. In this talk, we describe the use of a "nonchanneling" (off axis) signal to calibrate atomic resolution STEM EDXS and EELS images of strontium titanate [001] for the quantitative analysis. Figure 1 shows EDX concentration values obtained at specific atomic sites and averaged over the full image from a $61 \mathrm{~nm}$ thick sample. The effects of elastic and thermal scattering and the delocalization of the particular ionization edge can produce apparent number densities either larger or smaller than the actual values. By implementing an inversion process, the correct numbers could approximately be recovered [10].

\section{References}

[1] K. Kimoto et al., Nature 450 (2007) 702.

[2] D. A. Muller et al., Science 319 (2008) 1073.

[3] P. Wang et al., Phys. Rev. Lett. 101 (2008) 236102. 
[4] J.M. LeBeau et al., Phys. Rev. Lett. 100 (2008) 206101.

[5] J.M. LeBeau et al., Nano Lett. 10 (2010) 4405.

[6] H.S. von Harrach et al., Journal of Physics: Conference Series 241 (2010) 012015.

[7] A. Gubbens et al., Ultramicroscopy 110 (2010) 962.

[8] G. Kothleitner et al., Microsc. Microanal. doi 10.1017/S1431927614000130.

[9] B. D. Forbes et al., Phys. Rev. B 82 (2010) 104103.

[10] G. Kothleitner et al., Phys. Rev. Lett. (2014) accepted paper

[11] The substantial help of Bernd Kraus, Paul Thomas, and Ray Twesten from Gatan, as well as Meiken Falke and Ralf Terborg from Bruker and Dimitri Klenov from FEI, in setting up this particular analytical hardware configuration is acknowledged.
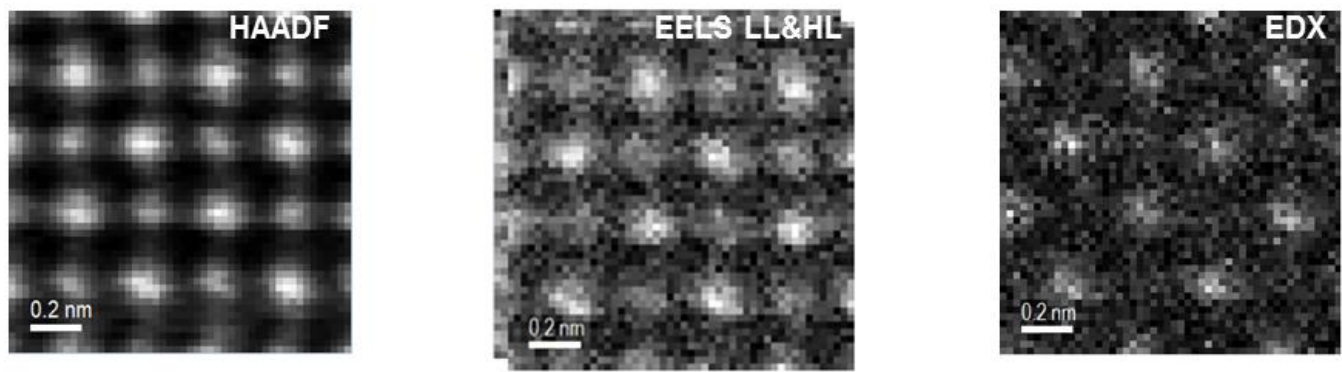

Figure 1. Strontium titanate [001] HAADF STEM image (left) taken simultaneously with low-loss and high-loss EELS (middle) and EDXS data (right) on a Quantum DualEELS electron spectrometer (Gatan) and a 0.7 sr Super-X x-ray detector (FEI).

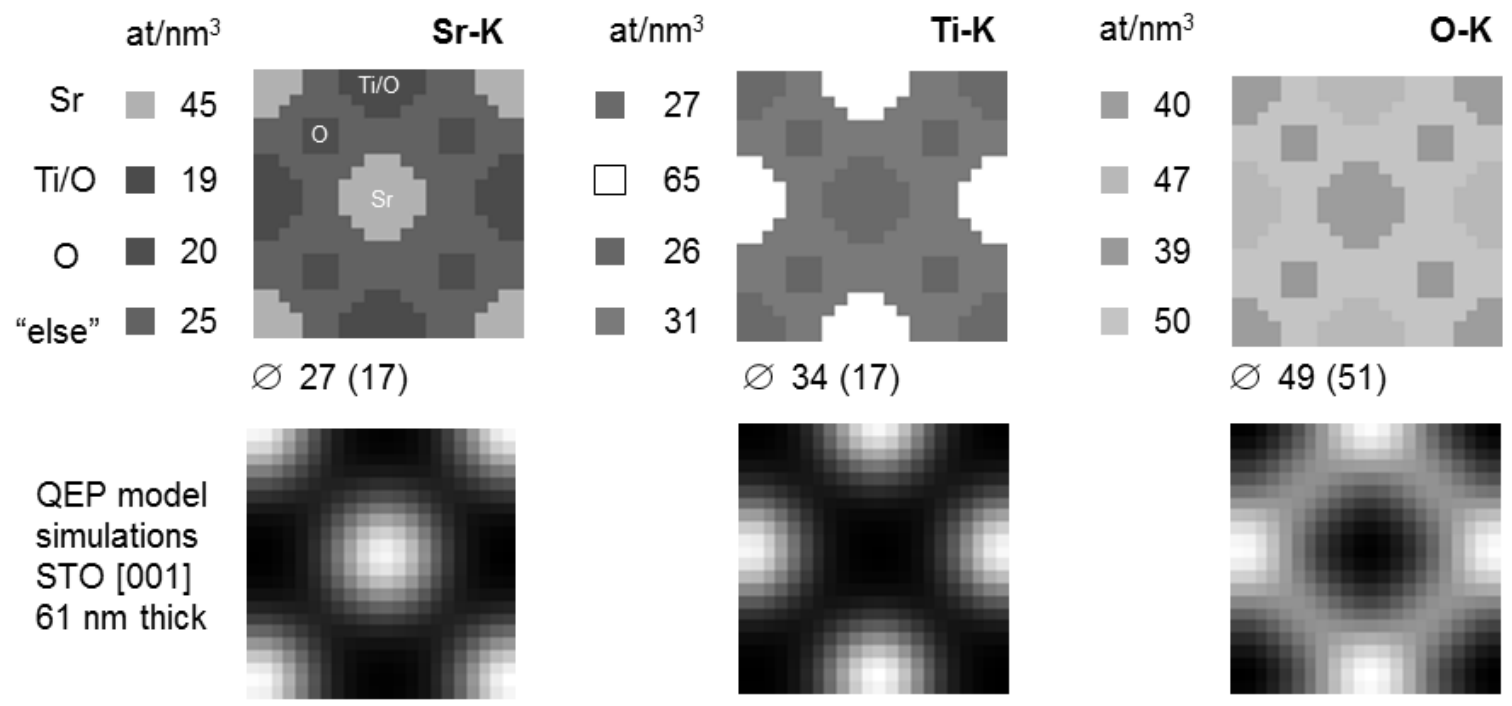

Figure 2. Absolute volumetric concentrations from EDXS data (upper row) together with QEP simulations (below); basis for an inversion [10]. 